\title{
Intelligent Terminal audio encoding research
}

\author{
Taoyang Luo ${ }^{123}$, Zou Long ${ }^{1,2,3},{ }^{*}$ Zhenrong Zhang ${ }^{123}$ \\ ${ }^{1}$ School of Computer, Electronics and Information, Guangxi University, Nanning 530004, China \\ ${ }^{2}$ Guangxi Key Laboratory of Multimedia Communications and Network Technology (Cultivating \\ Base), Guangxi University, Nanning 530004, China \\ ${ }^{3}$ Guangxi Colleges and Universities Key Laboratory of Multimedia Communications and Information \\ Processing, Guangxi University, Nanning 530004, China \\ ‘zzr76@gxu.edu.cn(Corresponding author)
}

Keywords.intelligent terminal,communication based on audio technology,Manchster-coded, 2FSK Abstract.According to the characteristics of our intelligent terminal audio port communication technology, Improving the communication done in-depth analysis and research on the coding efficiency.In HiJack platform to complete the 2 FSK codec and Manchester codec driver development and driver development based on the android operating system as well.And we tested these two communication encoding data communication performance between platforms and intelligent terminals.

\section{Introduction}

With the advent of intelligent terminal audio port communication technology, providing a new way of mentality and solutionsbyexpanding the sensor resources through the intelligent terminal audio port and external data to improve the ability of perceive intelligent terminals.

However, since the audio port is designed primarily for the transmission of an audio signal, the data can be processed at a frequency between $20 \mathrm{~Hz} \sim 20 \mathrm{KHz}$, and therefore less communication bandwidth, limiting the communication rate audio communication. Meanwhile, the audio port to transfer data are weak AC signal, the signal amplitude is generally less than $400 \mathrm{mV}$, susceptible to interference from external noise.

Hence the choice of a more efficient coding method, in order to ensure that resources are limited and under the audio ports ensure data transmission error rate as low as possible under the premise of effectively improve the data transfer rate, and make full use of the audio port output energy has important practical significance .

Since 2010 the University of Michigan has made Audio Hijack[1,2], platform architecture design has made some progress,for the first time proposed and implemented 2FSK modulation and Manchester encoding on the intelligent terminal and HiJackplatform. Therefore, this article focuses on two kinds of encoding, and to evaluate the use of three aspects of the data transmission rate, bit error rate, broadband.

\section{FSK codec test}

FSK (Frequency-Shift Keying)is the use of a different frequency carrier modulation scheme to transmit information[5], and two different frequencies represent logic 0 and a logic 1 to transmit data

We use intelligent terminal ZTE's V5, adopted $f_{1}=2 f_{2}$. Where $f_{1}$ represents logic 1 , logic 0 
indicates $f_{2}$. By increasing 2FSK-modulated carrier frequency to the limits, to test the recognition rate platform and intelligent terminal. According to the Nyquist sampling theorem, $44.1 \mathrm{KHz}$ sampling rate is theoretically possible to accurately identify the $22 \mathrm{KHz}$ signal.In order to ensure the symmetry of two-way communication and the quality of the data communication, we will set communication carrier $f_{1} 8192 \mathrm{~Hz}, 4096 \mathrm{~Hz}$ then the value of $f_{2}$

Next, weexamed the data transmission rate and bit error ratein 2 FSK modem based on communication platform and the intelligent terminal, We defined uplink is a platform, the intelligent terminal sends a defined data transmission to the mobile phone platform is defined as the downlink data, shown in Figure 1:

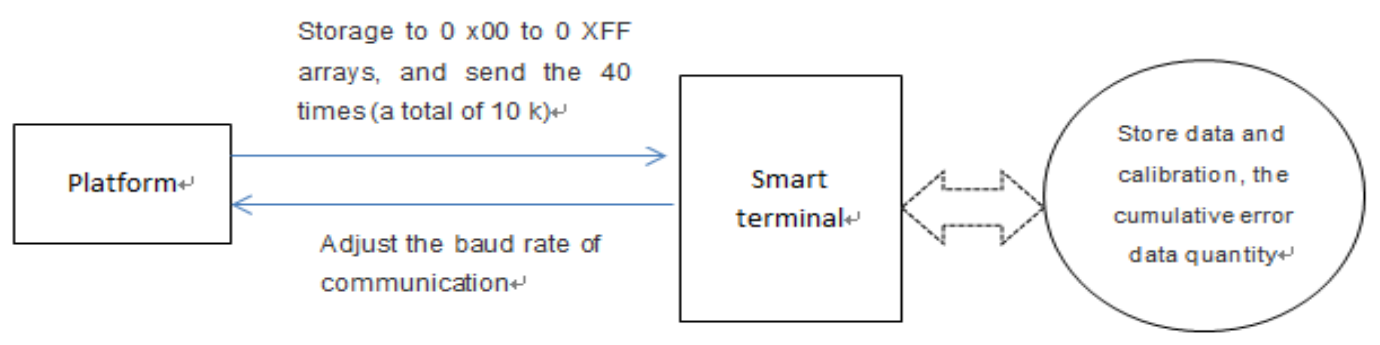

Fig.1Intelligent terminal and platform data transmission process

Test results as shown in Table1:

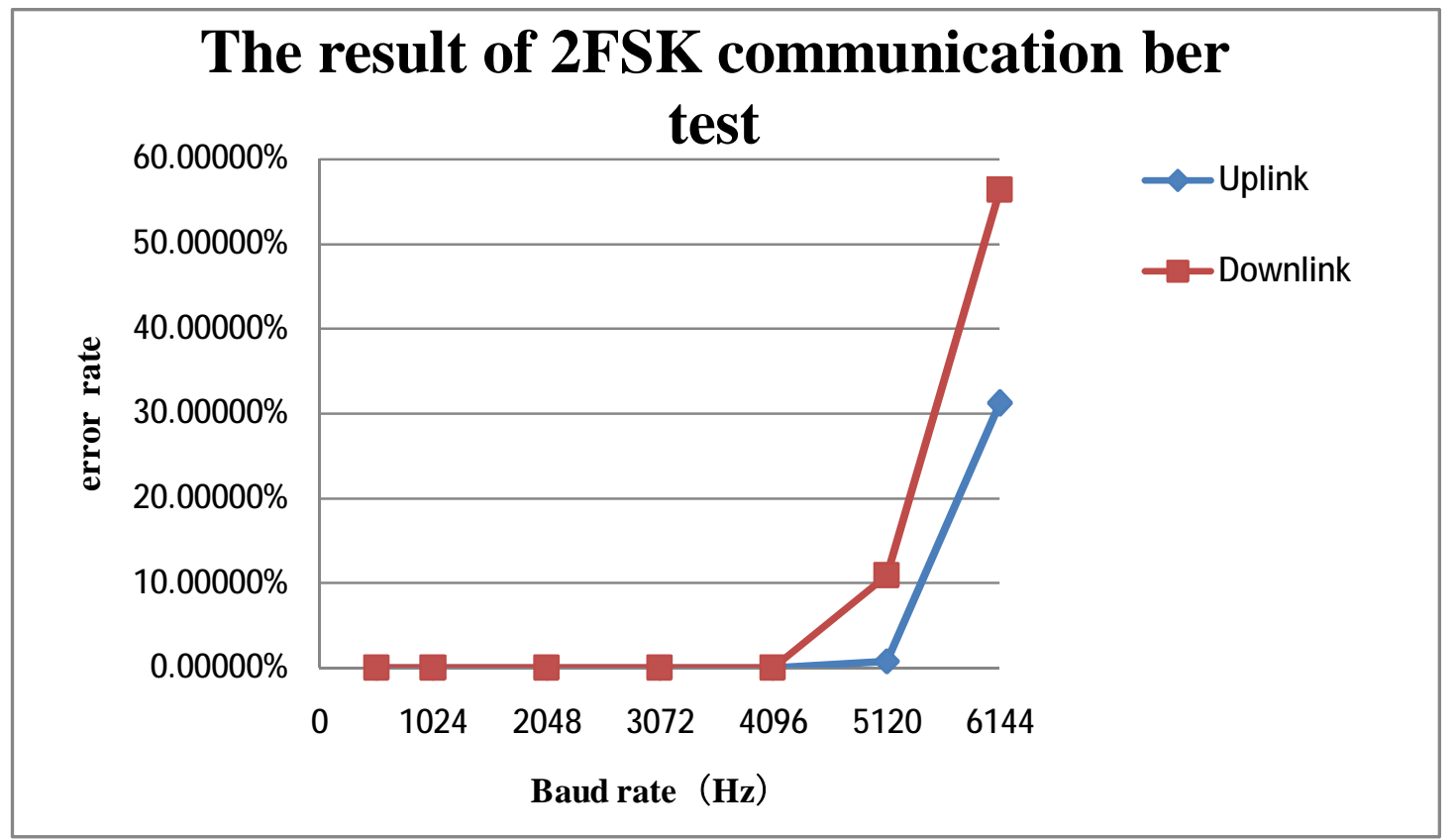

Table.1 The result of 2FSK communication ber test

2 FSK modulation technology in the baud rate is about $5000 \mathrm{hz}$ can obtain steady and reliable communication.

\section{Manchester encoding}

Manchester Encoding is a commonly used synchronous clock Encoding technology. It uses signal jump along the high and low level. We analysis the provisions in the IEEE 802.3 from low to high level jump presentation logic 1, with high - low level jump presentation logic 0 . And in order to 
realize data sampling must satisfy $f_{s} \geq 4 \times f_{1}$.

Since each bit corresponds to a jump, no dc component, the encoded signal is very suitable for ac signal transmission. Intelligent terminal on the audio signal for analog signal transmission, so the Manchester coding technique was used to realize data transmission is feasible[3].

Literature[4]test through the experiment the HiJack platform and intelligent terminal use Manchester coding performance of the communication. As the pace of baseband signal down to 8820 - bit/s, packet loss rate is the lowest.

On the software design, the two data transmission mode interrupt processing algorithm is encapsulated into a block of code, through a state identification to Manchester coding test mode, other steps like 2 FSK. Test result is shown in Table2.

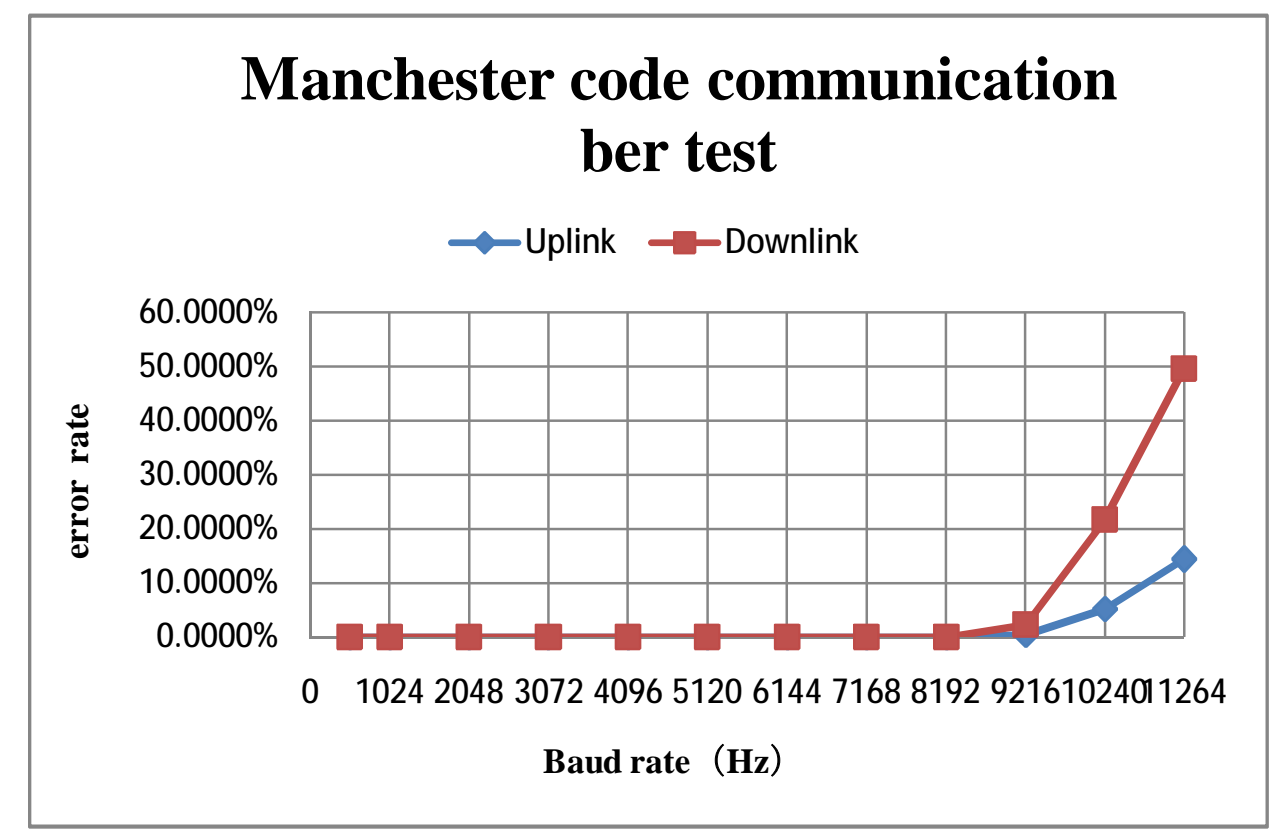

Table.2 Manchester code communication ber test

As Table 2 shows that proper baud rate is less than or equal to $9216 \mathrm{hz}$, the bit error rate in the normal communication within the acceptable range. Also verified in audio oral communication, when the signal frequency is about a quarter of sampling rate, can achieve a higher success rate of decoding.

\section{Performance analysis}

From the previous contrast can be found that Manchester coding is adopted to improve the communication, when the bit error rate in the acceptable range and 2 FSK communication frequency of $4096 \mathrm{~Hz}$, Manchester coding under the condition of baud rate to $8192 \mathrm{~Hz}$. The maximum communication rate is twice the 2 FSK communication.

Then we test two kinds of encoding for the maximum signal frequency $8192 \mathrm{~Hz}$ of $1 \mathrm{~m}$ data need time, each test three sets of data, the average as the final result. Test results as shown in figure 2. 


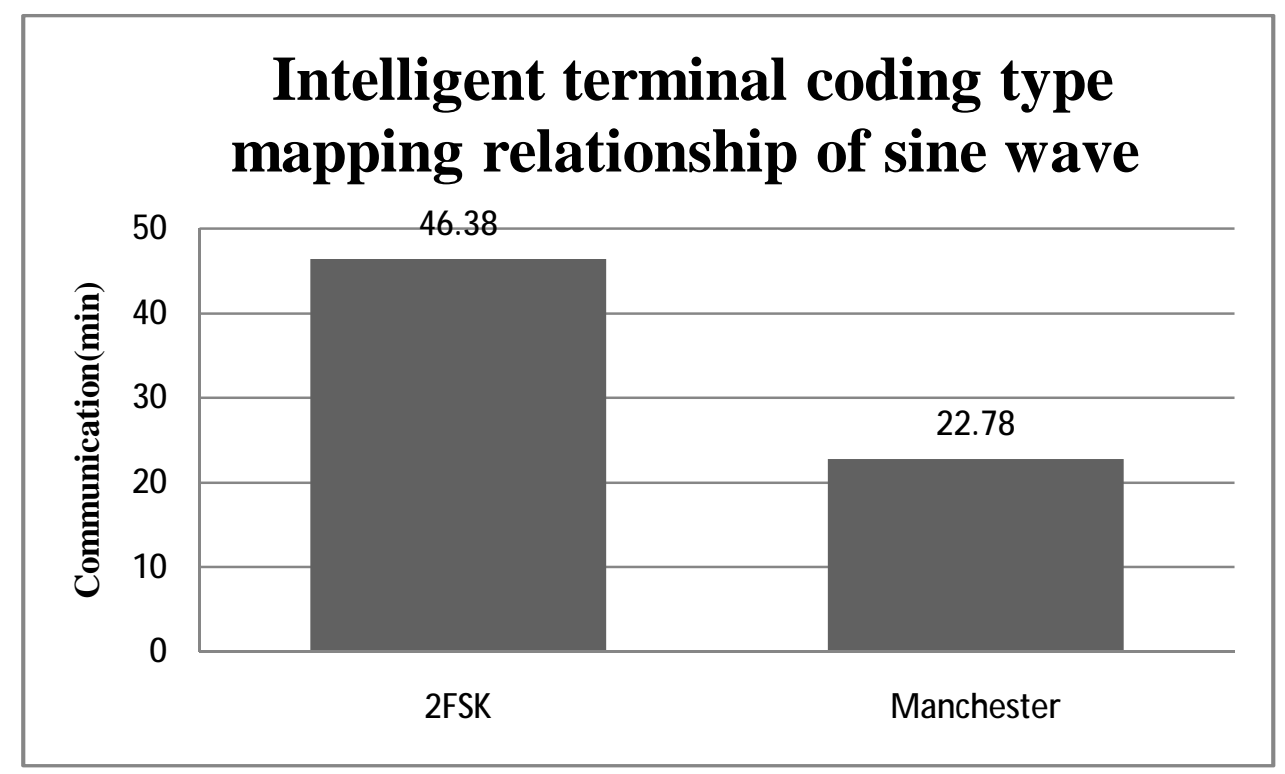

Fig.2 Intelligent terminal coding type mapping relationship of sine wave

From the figure 2,Manchester coding transmission rate also have advantages.

\section{Conclusions}

Based on the experimental platform to realize the 2 FSK and Manchester coding of these two kinds of communication coding mode, and test the two coding decoding way in ascending and descending rate and bit error rate of communication on the link. Two kinds of communication mode can meet the requirements of the low rate of data acquisition and transmission.But from the resources, make full use of audio frequency Manchester coding has a larger advantage.

\section{Acknowledgements}

Our works are supported by Natural Science Foundation of China (51407036) and Guangxi Science Foundation (2013GXNSFCA019019, 2014GXNSFBA118274) .

\section{References}

[1] Ye-Sheng Kuo, SonalVerma, Thomas Schmicl, Prabal Dutta. Hijacking Power and Bandwidth from the Mobile Phone's Audio Interfacc[C]. First Annual Symposium on Computing for Development(DEV' 10). New York,USA:ACM New York,USA,2010:24 .

[2] SonalVerma, Andrew Robinson, Prabal Dutta. AudioDAQ:Turning the Mobile Phone's Ubiquitous Headset Port into a Universal Data Acquisition Inlerface[C].Proceedings of the 10th ACM Conference on Embedded Networked Sensor Systems. New York,USA:ACM New York , USA, 2012:197-210.

[3] Chao L, Tsui C Y, Ki W H. A batteryless vibration-based energy harvesting system for ultra low power ubiquitous applications[C]//Circuits and Systems, 2007. ISCAS 2007. IEEE International Symposium on. IEEE, 2007: 1349-1352.

[4] Amiri I S, Ali J. Data signal processing via manchester coding-decoding method using chaotic signals generated by PANDA ring resonator[J]. Chinese Optics Letters, 2013, 11(4): 041901.

[5] Zhang J, Chi N, Holm-Nielsen P V, et al. Performance of Manchester-coded payload in an optical FSK labeling scheme[J]. IEEE Photonics Technology Letters, 2003, 15(8): 1174-1176. 